\title{
On extensions of Pascal's theorem
}

By H. W. Richmond, King's College, Cambridge.

(Received 12th August, 1936. Read 2nd November, 1936.)

1. The object of this paper is firstly to extend the theorem of Pascal concerning six points of a conic to sets of $2(n+1)$ points of the rational normal curve of order $n$ in space of $n$ dimensions; secondly to explain why a wider extension to other sets of $2(n+1)$ points in $[n]$ must be sought; and lastly to give briefly an extension to [3] and [4] which will be further generalised in a later paper. The striking feature of Pascal's theorem-that each of the sixty ways of arranging the points in a cycle, or as vertices of a closed polygon, leads to a different version of the theorem-is retained in the following extension to $[n]$.

If $2(n+1)$ points of a rational normal $C_{n}$ in [n] are taken as the vertices of a polygon, the $[n-2]$ 's which are determined each as the intersection of one of the $n+1$ pairs of opposite primes of the polygon have the property that every line which intersects all but one of these [n-2]'s must also intersect the last.

To establish this the procedure is the same for all values of $n$; proofs for the values 3 and 4 of $n$ will explain it.

2. In space of three dimensions, if eight points $P_{1}, P_{2}, P_{3}, P_{4}, P_{5}$, $P_{6}, P_{7}, P_{8}$ of a twisted cubic curve are taken as vertices of a skew polygon of eight sides, the four lines of intersection of opposite planes of the polygon, $\left(P_{1} P_{2} P_{3}\right.$ and $P_{5} P_{6} P_{7} ; \quad P_{2} P_{3} P_{4}$ and $P_{6} P_{7} P_{8}$; $P_{3} P_{4} P_{5}$ and $P_{7} P_{8} P_{1} ; P_{4} P_{5} P_{6}$ and $\left.P_{8} P_{1} P_{2}\right)$ will be generators of a hyperboloid.

It is clear that, if $a_{1}, a_{2}, a_{3}, a_{4}, a_{5}, a_{6}, a_{7}, a_{8}$ denote any eight numbers, then

$$
\begin{aligned}
& \left(a_{1}-a_{5}\right)\left(a_{2} a_{3} a_{4}-a_{6} a_{7} a_{8}\right)+\left(a_{2}-a_{6}\right)\left(a_{3} a_{4} a_{5}-a_{7} a_{8} a_{1}\right) \\
& \quad+\left(a_{3}-a_{7}\right)\left(a_{4} a_{5} a_{6}-a_{8} a_{1} a_{2}\right)+\left(a_{4}-a_{8}\right)\left(a_{5} a_{6} a_{7}-a_{1} a_{2} a_{3}\right)=0 .
\end{aligned}
$$

Replace $a_{n}$ by $\left(\theta-e_{n}\right) /\left(\phi-e_{n}\right)$, and clear away the fractions by multiplying by the product of all the quantities $\left(\phi-e_{n}\right)$. The first factor of the first term, $a_{1}-a_{5}$, gives us

$$
\left(\theta-e_{1}\right)\left(\phi-e_{5}\right)-\left(\theta-e_{5}\right)\left(\phi-e_{1}\right)=\left(e_{1}-e_{5}\right)(\theta-\phi) .
$$


When the common factor $(\theta-\phi)$ is rejected, the first factor in each term is reduced to a constant, $e_{1}-e_{5}, e_{2}-e_{6}, e_{3}-e_{7}$, or $e_{4}-e_{8}$.

The second factor of the first term, $a_{2} a_{3} a_{4}-a_{6} a_{7} a_{8}$, gives us

$$
\left|\begin{array}{ll}
\left(\theta-e_{2}\right)\left(\theta-e_{3}^{*}\right)\left(\theta-e_{4}\right), & \left(\theta-e_{6}\right)\left(\theta-e_{7}\right)\left(\theta-e_{8}\right) \\
\left(\phi-e_{2}\right)\left(\phi-e_{3}\right)\left(\phi-e_{4}\right), & \left(\phi-e_{6}\right)\left(\phi-e_{7}\right)\left(\phi-e_{8}\right)
\end{array}\right|
$$

or, when the determinant is expanded, six terms $\left(\theta^{r} \phi^{8}-\theta^{s} \phi^{r}\right)(r$ and $\delta$ having values $0,1,2$ or 3 ), each term with a coefficient made up from the numbers $e_{n}$. But, if $e_{1}, e_{2}, \ldots e_{7}, e_{8}$ are values of the parameter $\theta$ which correspond to eight points $P_{1}, P_{2}, \ldots P_{7}, P_{8}$ of the twisted cubic curve

$$
x_{0}: x_{1}: x_{2}: x_{3}:: \theta^{3}: \theta^{2}: \theta: 1 \text {, }
$$

these coefficients are seen to be the six coordinates of the line of intersection of the planes $P_{2} P_{3} P_{4}$ and $P_{6} P_{7} P_{8}$. Thus we have four lines, each determined as the intersection of two planes; and, if we take any one of the six coordinates of each line and multiply by the respective constants

$$
e_{1}-e_{5}, \quad e_{2}-e_{6}, \quad e_{3}-e_{7}, e_{4}-e_{8}
$$

the sum vanishes. This proves that a linear complex which contains three of the lines must also contain the fourth, or that any line which meets three of the lines must also meet the fourth, or that the four lines are generators (of the same system) of a hyperboloid.

3. In the same way, if we take ten numbers $a_{1}, a_{2}, \ldots a_{8}, a_{9}, a_{10}$, the sum of five expressions such as

$$
\left(a_{1}-a_{6}\right)\left(a_{2} a_{3} a_{4} a_{5}-a_{7} a_{8} a_{9} a_{10}\right)
$$

is seen to vanish. When we replace $a_{n}$ as before by $\left(\theta-e_{n}\right) /\left(\phi-e_{n}\right)$ and clear away the fractions, the first factor is reduced to the constant $e_{1}-e_{6}$. The second factor gives us a determinant of two rows as before, quartic functions of $\theta$ and $\phi$ taking the places of cubic functions. When we consider the points $P_{1}, P_{2}, \ldots P_{8}, P_{9}, P_{10}$ on the rational normal $C_{4}$ in [4],

$$
x_{0}: x_{1}: x_{2}: x_{3}: x_{4}:: \theta^{4}: \theta^{3}: \theta^{2}: \theta: 1,
$$

which correspond to the values $e_{1}, e_{2}, \ldots e_{8}, e_{9}, e_{10}$ of $\theta$, we see that the functions of the numbers $e_{n}$ which appear as coefficients in the determinant are the ten coordinates of the plane common to the primes $P_{2} P_{3} P_{4} P_{5}$ and $P_{7} P_{8} P_{9} P_{10}$. As before, the fact that this determinant multiplied by $e_{1}-e_{6}$ and the four similar products 
(obtained by moving the suffixes forward cyclically in their natural order) have zero sum proves that every line which intersects four of these planes must intersect the fifth also. The planes form a set of five associated planes in [4]. Hence we have the theorem:-

In [4], if ten points of a rational normal quartic curve are taken as vertices of a polygon of ten sides, the five pairs of opposite primes of the polygon intersect in five planes which form a set of five associated planes.

Exactly similar reasoning proves the theorem enunciated in $\S 1$ for any other value of $n$. In $[n]$, a $[n-2]$ is the dual of a line; and, just as a line is defined by $\frac{1}{2} n(n+1)$ coordinates, each a determinant of two rows in the coordinates of two points of the line, so a [n-2] is defined by $\frac{1}{2} n(n+1)$ coordinates, each a determinant of two rows in the coefficients of the equations of two primes through the $[n-2]$. That a line and a $[n-2]$ should intersect, i.e. should have one common point and lie in a prime, imposes one condition upon the coordinates of the line and the $[n-2]$, linear in the coordinates of each.

4. Starting from a set of points on a curve, we have been led to a system of [ $n-2]$ 's possessing a certain property. Can the process be reversed? Can we for example, working in space of three dimensions, start from four generators of a hyperboloid and work back to a skew octagon whose opposite planes intersect in the generators, and will the eight vertices of the octagon lie upon a twisted cubic curve? For ordinary space the answer to the questions is known.

In Vol. 12 of Acta Mathematica there are papers by Dobriner (pp. 339-361) and Zeuthen (pp. 362-366) dealing with the eight points common to three quadrics, and in the second paper Zeuthen proves that these eight points possess the property that we have proved in $\S 2$ for any eight points of a twisted cubic curve. A geometrical proof by Schroeter will be found in Vol. 14 (pp. 207-209). But Prof. H. F. Baker has drawn my attention to a very remarkable paper published in 1850 in Vol. 5 of the Cambridge and Dublin Mathematical Journal (pp. 58-69), by T. Weddle, who not only anticipated Zeuthen's discovery by 35 years but at the same time established the converse theorem. Given four generators of a hyperboloid, 1, 2, 3, 4, in order to derive a skew octagon we take any two planes through each generator, $P_{1}, Q_{1}$ through 1 , and so on. We 
arrange these planes in a cycle, e.g. $P_{1} P_{2} P_{3} P_{4} Q_{1} Q_{2} Q_{3} Q_{4} P_{1} P_{2}$ so that the two through a generator are opposite. Then each two consecutive planes in the cycle intersect in a side, and each three consecutive planes intersect in a vertex of the octagon. The eight vertices, Weddle shows, are the common points of three quadrics. Exceptionally they will lie upon a twisted cubic curve, but they will not generally do so.

Thus it appears that, although the theorems concerning $2(n+1)$ points of a $C_{n}$ in $[n]$ lend themselves readily to extension to space of any number of dimensions, they do not fully illustrate the properties of the configurations which they lead us to study. Algebraic methods of proof are greatly simplified when the parametric expressions for the coordinates of points of a $C_{n}$ are available for our use; without them algebraic methods tend to become laborious. But certain results may be obtained by geometrical reasoning and are of importance. A certain well-known figure will be found useful.

Place six points $A, Z, B, X, C, Y$, round an oval and join $A B$, $B C, C Z, Z Y, Y X, X A$. Let $B C$ and $Y X$ meet in $U, C Z$ and $A X$ in $V, Z Y$ and $A B$ in $W$. By Pascal's theorem, if $A, B, C, X, Y, Z$ lie on a conic, $U, V, W$ lie on a line; conversely, if $U, V, W$ are collinear, $A, B, C, X, Y, Z$ lie on a conic; moreover the pencils of lines $X(A B C Y)$ and $Z(A B C Y)$ have equal cross-ratios.

5. To prove Weddle's theorem in space of three dimensions, let $1,2,3,4$ be four lines such that every transversal that meets three of them must also meet the fourth line; and let $P_{1}, P_{2}, P_{3}, P_{4}$, $Q_{1}, Q_{2}, Q_{3}, Q_{4}$, be eight planes, each passing through the line denoted by the suffix. Taken in this order these planes determine a skew polygon of eight sides and eight vertices whose pairs of opposite planes intersect in the lines $1,2,3,4$. Denote the vertices of the octagon by $A, B, C, D, E, F, G, H, A, B, C$ lying in $P_{1}, B, C, D$ in $P_{2}$, $C, D, E$ in $P_{3}$, and so on.

Consider the figure in the plane $P_{1}$ in connection with the figure described above. $A, R, C$ are in the plane; we suppose that $Z$ and $X$ are the points where $D E$ and $G H$ respectively meet the plane. Then $B C, C Z, B A, A X$ are the lines in which $P_{1}$ is met by $P_{2}, P_{3}, Q_{4}, Q_{3}$. Again $P_{4}$ or $D E F$ and $Q_{2}$ or $F G H$ meet $P_{1}$ in two lines through $Z$ and $X$ respectively; we suppose them to be $Z Y$ and $X Y$. Then, since $U$ lies on $P_{2}$ and $Q_{2}, V$ on $P_{3}$ and $Q_{3}, W$ on $P_{4}$ and $Q_{4}, U, V, W$ are the points where the lines 2,3,4 meet the plane $P_{1}$; the fourth line 1 lies 
wholly in the plane. Since the line $U V$ meets the first three of the lines $1,2,3,4$ it must also meet the last, i.e. $U, V, W$ must be collinear, $A, B, C, X, Y, Z$ must lie on a conic and the four planes through $D E$ and $A, B, C, Y$ must have the same anharmonic ratio as those through $G H$ and the same four points. That is to say

$$
D E(A, B, C ; F)=G H(A, B, C, F) .
$$

It follows that there is a quadric on which lie the lines $D E$ and $G H$ and the points $A, B, C, F$. Each of the planes $P_{n}$ and $Q_{n}$ provides a quadric such as this passing through the eight points $A, B, C, D, E$, $F, G, H$,-eight quadrics in all.

Conversely we may start (as Schroeter does) from a set of eight points common to three quadrics and therefore to a net of quadrics. If the points, in order, are $A, B, C, D, E, F, G, H$, one quadric of the net has the lines $D E$ and $G H$ as generators, and Pascal's theorem applied to the section of this quadric by the plane $A B C$ proves that the four lines of intersection of opposite planes of the octagon $A B C D E F G H$ are all met by a line in the plane $A B C$. Similarly it is shown that they are all met by a line in the plane $B C D$, etc., etc., i.e. by eight lines in all. It is proved beyond doubt that the four lines belong to a regulus and that the eight points in the preceding paragraph are common to a net of quadrics.

6. In space of four or more dimensions similar results are obtained, but it is not possible to draw conclusions so simply. We may confine ourselves to [4]. The analogue of the four lines is the well-known system of five associated planes, which will be denoted by $1,2,3,4,5$ : two primes pass through each plane, $P_{1}$ and $Q_{1}$ through $1, P_{2}$ and $Q_{2}$ through 2 , and so on. Arranged in cyclical order

$$
P_{1}, P_{2}, P_{3}, P_{4}, P_{5}, Q_{1}, Q_{2}, Q_{3}, Q_{4}, Q_{5}
$$

they generate a polygon of ten sides and ten vertices, each vertex being the point common to four successive primes. The vertices may be named $A, B, C, D, E, F, G, H, J, K, A$ lying in the primes $P_{1}, P_{2}, P_{3}, P_{4}, B$ in $P_{2}, P_{3}, P_{4}, P_{5}$, and so on. Four consecutive vertices lie in each prime, e.g. $A, B, C, D$ in $P_{4}$; pairs of opposite primes intersect in the five associated planes, e.g. the primes $A B C D$ and $F G H J$, or $P_{4}$ and $Q_{4}$, intersect in the plane 4 .

As before consider the figure in the plane $A B C$, in connection with the figure described at the end of $\$ 4$. The points $A, B, C$ are shown in that figure; the points $Z$ and $X$ are the points in which the 
planes $D E F$ and $H J K$ meet $A B C$, for in [4] two planes have one common point; the lines $Z Y$ and $X Y$ are the lines in which the primes $D E F G$ and $G H J K$ respectively meet $A B C$.

The lines $A B, B C, C Z, Z Y, Y X, X A$ are the lines of intersection of $A B C$ with the primes $J K A B, B C D E, C D E F, D E F G$, GHJK, $H J K A$, or $P_{4}, P_{5}, Q_{1}, Q_{2}, Q_{5}, P_{1}$. Thus $U$, lying in $Q_{5}$ and $P_{5}$, is the point in which $A B C$ meets the plane 5; similarly $V$ and $W$. are the points in which $A B C$ meets 1 and 2. As to the other two of the five associated planes, 3 and 4, $A B C$ lies wholly in the primes $P_{3}$ and $P_{4} ; A B C$ meets 3 and 4 in the lines where it intersects the primes $Q_{3}$ and $Q_{4}$, so that every line in $A B C$ meets 3 and 4 . Hence $U V$ meets $1,3,4,5$, four of the five associated planes, and must meet the fifth also. As in $\S 5$, $U, V, W$ must be collinear, and $A, B, C, X, Y, Z$, must lie upon a conic; the four primes which pass through the plane $D E F$ and the four points $A, B, C, G$ must have the same anharmonic ratios as the four through $H J K$ and these points. Two projective pencils of primes through $D E F$ and $H J K$ generate a quadric passing through $A, B, C$ and $G$. The same reasoning can be applied to each of the planes $B C D, C D E, \ldots$, so we have ten quadrics passing through the ten points $A, B, C, D, E, F, G, H, J, K$. Each quadric is a cone; the point common to $D E F$ and $H J K$ is the vertex of the one first discussed.

Conversely, it is known that in [4] there exist sets of ten points having the property that every quadric passing through nine of them must pass through the tenth point ${ }^{1}$. Starting from such a set of points, $A, B, C, D, E, F, G, H, J, K$, we observe that whereas in general fourteen points determine a quadric, yet a quadric can be made to pass through these ten and five other points. Take three points in the plane $D E F$ and two in $H J K$. The quadric passes through six points of the plane $D E F$ and must contain the whole plane; further, since $D E F$ and $H J K$ have one common point, it passes through six points in $H J K$ and must contain the whole of $H J K$ also. By the argument of the previous section we prove that a line in the plane $A B C$ meets each of the five planes of intersection of opposite primes of the decagon $A B C D E F G H J K$; and in the same way we prove that ten lines meet each of these planes.

But these conditions unfortunately do not constitute a proof. It is highly probable, but not certain, that the ten points obtained in the

1 See the Encyllopaedie d. math. Wissenschaften, Bd. III, 2.2. A., pp. 836, 837. 
first part of this section are a set of ten associated points: to prove it we must consider how many of the ten quadrics are linearly independent. As regards the final paragraph, since an infinity of lines meet any five planes, to show that ten lines meet five particular planes proves nothing without further investigation. But a different line of reasoning establishes the result directly ${ }^{1}$.

7. As in $\S 5$, take eight associated points in [3], $A, B, C, D, E, F$. $G, H$, i.e. eight points common to three quadries, and denote the planes

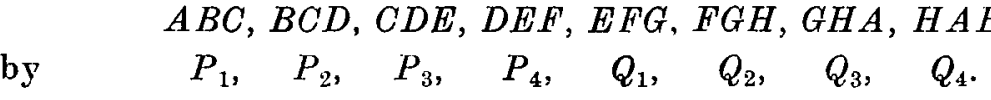

Consider the tetrahedron formed by $P_{1}, P_{2}, P_{3}, P_{4}$, denoting its vertices by $X_{1}, X_{2}, X_{3}, X_{4} ; X_{1}$, being common to the planes $B C D$, $C D E, D E F$, coincides with $D$, and $X_{4}$, being common to $A B C, B C D$, $C D E$, coincides with $C$. Now it is a fundamental property of eight associated points that, if they are separated into two groups of four the two tetrahedra thus obtained are both self-polar with respect to a certain quadric. Thus there is a quadric $\Sigma$ with respect to which the tetrahedra $H A B C$ and $D E F G$ are both self-polar. What are the polar planes of $X_{1}, X_{2}, X_{3}, X_{4}$ with respect to $\Sigma$ ? That of $X_{1}$ or $D$ is $E F G$ or $Q_{1}$; that of $X_{4}$ or $C$ is $H A B$ or $Q_{4}$. $X_{2}$ is the point common to $P_{1}, P_{3}, P_{4}$, i.e. to the plane $A B C$ and the line $D E$; its polar with respect to $\Sigma$ is therefore the plane containing the point $H$ and the line $F G$, i.e. the plane $F G H$ or $Q_{2} . \quad X_{3}$ is the point common to $A B C, B C D, D E F$, i.e. to the line $B C$ and the plane $D E F$; its polar plane therefore contains the line $H A$ and the point $G$, i.e. is the plane $G H A$ or $Q_{3}$.

$P_{1}, P_{2}, P_{3}, P_{4}$ are the faces of a tetrahedron and $Q_{1}, Q_{2}, Q_{3}, Q_{4}$ are the polar planes of its vertices with respect to a certain quadric $\Sigma$. If the equation of $\Sigma$ in coordinates $x_{1}, x_{2}, x_{3}, x_{4}$ referred to this tetrahedron $X_{1} X_{2} X_{3} X_{4}$ is

$$
c_{11} x_{1}^{2}+c_{22} x_{2}^{2}+\ldots+2 c_{12} x_{1} x_{2}+\ldots+2 c_{34} x_{3} x_{4}=0,
$$

the equation of the plane $Q_{1}$ is

$$
c_{11} x_{1}+c_{12} x_{2}+c_{13} x_{3}+c_{14} x_{4}=0
$$

$1 \$$ 1-6 of this paper are the outcome of work carried on at intervals during several years. The method of $\$ \S 7$ and 8 was discovered only after the former sections were already in type. 
The six coordinates $q_{12}, q_{13}, q_{14}, q_{34}, q_{24}, q_{23}$, of the line of intersection of $Q_{1}$ and $P_{1}$, and of the three similar lines with suffix 2,3 or 4 are

$\begin{array}{cccccc}c_{12} & c_{13} & c_{14} & 0 & 0 & 0 \\ -c_{12} & 0 & 0 & 0 & c_{24} & c_{23} \\ 0 & -c_{13} & 0 & c_{34} & 0 & -c_{23} \\ 0 & 0 & -c_{14} & -c_{34} & -c_{24} & 0 .\end{array}$

Since the sum of the numbers in each column vanishes, it follows that any linear complex which contains three of the lines must contain the fourth line.

8. Again, as in $\S 6$, take $A, B, C, D, E, F, G, H, J, K$, ten associated points in [4], and denote the primes

by $A B C D, B C D E, C D E F, D E F G, E F G H$, and $F G H J, G H J K, H J K A, J K A B, K A B C$,

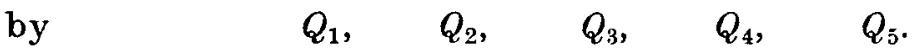

The vertices of the simplex formed by $P_{1}, P_{2}, P_{3}, P_{4}, P_{5}$ are denoted by $X_{1}, X_{2}, X_{3}, X_{4}, X_{5} ; X_{1}$ now coincides with $E$ and $X_{5}$ with $D$. Again there is a quadric $\Sigma$ such that both of the simplexes $K A B C D$ and $E F G H J$ are self-polar with respect to it, and again it appears that $Q_{1}, Q_{2}, Q_{3}, Q_{4}, Q_{5}$ are the polar primes of $X_{1}, X_{2}, X_{3}, X_{4}, X_{5}$, with respect to $\Sigma$. For, firstly, the polar primes of $X_{1}$ and $X_{5}$, i.e. $E$ and $D$, are $F G H J$ or $Q_{1}$ and $K A B C$ or $Q_{5}$. Nextly, $X_{2}$, being common to the primes $P_{1}, P_{3}, P_{4}, P_{5}$, lies on the prime $A B C D$ and the line $E F$. Its polar prime with respect to $\Sigma$ therefore contains the point $K$ and the plane $G H J$, i.e. in $Q_{2}$. In the same way the polar prime of $X_{4}$ is $Q_{4}$. Lastly, $X_{3}$ being common to the primes $P_{1}, P_{2}, P_{4}, P_{5}$, lies in the planes $B C D$ and $E F G$; its polar prime therefore contains the lines $K A$ and $H J$ and is $Q_{3}$.

Thus the primes $P_{1}, P_{2}, P_{3}, P_{4}, P_{5}$ form a simplex and $Q_{1}, Q_{2}$, $Q_{3}, Q_{4}, Q_{5}$ are the polar primes of its vertices with respect to a certain quadric $\Sigma$. The argument used in $\S 7$ with the necessary modifications proves the planes of intersection of $P_{1} Q_{1}, P_{2} Q_{2}, P_{3} Q_{3}, P_{4} Q_{4}$, $P_{5} Q_{5}$ to be five associated planes. 